\title{
Correction to: Editorial: Special Issue on Heart Valve Mechanobiology
}

\section{New Insights into Mechanical Regulation of Valve Disease and Regeneration}

\author{
Craig A. Simmons ${ }^{1,2,3}$ and Hanjoong Jo $\mathbf{J}^{4,5}$ \\ ${ }^{1}$ Translational Biology and Engineering Program, Ted Rogers Centre for Heart Research, Toronto, ON, Canada; ${ }^{2}$ Institute of \\ Biomaterials and Biomedical Engineering, University of Toronto, Toronto, ON, Canada; ${ }^{3}$ Department of Mechanical and \\ Industrial Engineering, University of Toronto, Toronto, ON, Canada; ${ }^{4}$ Coulter Department of Biomedical Engineering, Emory \\ University and Georgia Institute of Technology, Atlanta, GA, USA; and ${ }^{5}$ Department of Cardiology, Emory University, \\ Atlanta, GA, USA
}

(Published online 24 May 2018)

\section{Correction to: \\ Cardiovascular Engineering and Technology (2018) https://doi.org/10.1007/s13239-018-0360-3}

Original article has been updated to include names and affiliations of authors. Due to error in production process, PDF version of original article did not include author names and affiliations.

Address correspondence to Craig A. Simmons, Department of Mechanical and Industrial Engineering, University of Toronto, Toronto, ON, Canada and Hanjoong Jo, Coulter Department of Biomedical Engineering, Emory University and Georgia Institute of Technology, Atlanta, GA, USA. Electronic mails: c.simmons@utoronto.ca, hjo@bme.gatech.edu

The original article can be found online at https://doi.org/10. 1007/s13239-018-0360-3. 